\title{
Impairment-Aware Design of Translucent DWDM Networks Based on the $k$-Path Connectivity Graph
}

\author{
Giuseppe Rizzelli, Massimo Tornatore, Guido Maier, and Achille Pattavina
}

\begin{abstract}
In this paper we propose a design procedure based on comprehensive integer linear programing (ILP) for translucent optical transport networks (OTNs), exploiting an extended version of the so-called "connectivity graph." For the first time to the best of our knowledge, we propose a mathematical approach that covers the manifold challenges of a realistic optical layer OTN design, jointly solving the regenerator placement problem (RPP) and the routing, fiber and wavelength assignment with regenerator problem (RFWA-RP). As a first contribution, we extend the concept of the connectivity graph to a $k$-path $(k-p)$ connectivity graph. Second, the formulation addresses the problem of planning the number of dense wavelength division multiplexing systems, jointly solving the RPP and placing transponders. Third, we consider regeneration devices as wavelength converters also, showing the benefits arising from that aspect disregarded in the existing impairmentaware (IA) ILP formulations based on the connectivity graph. Finally we compare over different networks the results of our design procedure with those achieved by a hybrid method that combines a simplified IA-ILP formulation with a greedy heuristic. Moreover, an analytical framework to evaluate the network cost in terms of capital expenditure and operational expenditure is also presented. We show that the $k-p$ connectivity graph represents a cost-effective tool for network design, as it allows one to greatly reduce the number of resources needed in both the protected and the unprotected scenarios.
\end{abstract}

Index Terms-Connectivity graph; Impairment-aware integer linear programing (IA-ILP) formulation; Regenerator placement problem (RPP); Routing fiber and wavelength assignment with regenerator problem (RFWA-RP); Translucent network design.

\section{INTRODUCTION}

D emand for higher bandwidth at lower cost is steadily increasing in today's communication networks. Ultrahigh-definition video, 3D Internet, 3D multimedia, multimedia-supported social networking, and many other broadband services are pushing service providers and equipment vendors towards new and improved solutions for scalable optical networks. One of the major limitations to scalability is the cost of optical-electrical-optical (O-E-O) interfaces (also known as transponders, TXPs) deployed in the network. A connection undergoes $\mathrm{O}-\mathrm{E}-\mathrm{O}$ conversion both to regenerate signal (3R: reamplification, reshaping, and retiming) and to change the wavelength. O-E-O converters are expen-

Manuscript received August 3, 2011; revised March 19, 2012; accepted March 22, 2012; published April 16, 2012 (Doc. ID 152331).

Giuseppe Rizzelli (e-mail: rizzelli@elet. polimi.it), Massimo Tornatore, Guido Maier, and Achille Pattavina are with CNIT, Italy, and are also with the Department of Electronics and Information, Politecnico di Milano, Via Ponzio 34-35, 20121 Milan, Italy.

Digital Object Identifier 10.1364/JOCN.4.000356 sive devices and consume a lot of power. Thus network operators recently started exploiting the optical-bypass technique: a connection remains in the optical domain as long as possible by transparently passing through nodes such as directionless/colorless/contentionless reconfigurable optical add-drop multiplexers (ROADMs) or the optical cross connects (OXCs) [1,2]. Different kinds of optical transport networks (OTNs) have been identified according to the utilization of O-E-O devices: opaque, transparent, and translucent $[3,4]$.

An opaque OTN is equipped by $\mathrm{O}-\mathrm{E}-\mathrm{O}$ interfaces, one for every wavelength, at both end nodes of each link of the network; this approach simplifies network management, design and control, as it implies a full independence of the logical layer from the physical layer. On the other hand, it is expensive and power consuming.

The opposite case is the transparent OTN in which O-E-O conversions do not occur in intermediate nodes, which are optically bypassed by signals. With optical transparent switching, OTN design and operation become cross-layer problems coupling the physical to the logical layer: transmission impairments and wavelength assignment have to be taken into account when planning the network and assigning resources to the lightpaths. Eliminating $\mathrm{O}-\mathrm{E}-\mathrm{O}$ conversions completely at intermediate nodes is possible only for limited-size networks, because transmission impairments impose a maximum distance reachable from the source node.

In the translucent approach both the opaque and the transparent features coexist in a node: TXPs are deployed only where necessary; i.e., an $\mathrm{O}-\mathrm{E}-\mathrm{O}$ operation is performed only if the signal quality falls below a certain threshold or $\lambda$ conversion is needed to avoid wavelength blocking [5-7]. The translucent approach is applicable to networks of any size; thus translucent network design may be regarded as a "green" future-proof solution for core networks [8].

Our aim for the study in this paper is to propose a new and improved single-layer translucent OTN design technique. One of the most promising of such techniques is the connectivity graph [9]. We enhance this technique, extending it to the $k$-path $(k-p)$ connectivity graph, so that our impairment-aware (IA) procedure can choose among $k$-paths for each logical edge instead of considering just the shortest path on the physical graph. We will show that increasing the value of $k$ makes it possible to improve the performance in terms of cost or power-consumption minimization. We present an IA-integer linear programing (ILP) formulation modeling the $k-p$ connectivity graph, and we show how to easily extend it to the path-protection case. Moreover, our method offers the 
following features included for the first time, to the best of our knowledge, in IA-ILP methods based on the connectivity graph: (a) TXPs can be used as both regenerators and wavelength converters (this opportunity, often explored in the existing heuristic approaches, was never captured by previous mathematical models based on the connectivity graph); (b) a comprehensive set of physical layer impairments is taken into account; (c) the number of dense wavelength division multiplexing (DWDM) systems in the translucent OTN is not preassigned, but rather minimized; (d) the number of TXPs is not preassigned, but computed; (e) it prevents a path on the connectivity graph from being composed of partially overlapping subpaths, which is a common problem when logical graphs are exploited for network design. Moreover, we show how to extend this formulation to encompass some practical deployment constraints. Besides, we also propose a hybrid ILP/heuristic procedure [10] applicable to large size design problems for which IA-ILP is not computationally practicable.

It has to be specified that our OTN model does not account for client-layer processing (e.g., traffic grooming), as it is outside the purpose of this paper. Moreover, for one specific class of impairments we resort to a worst-case approach (i.e., the impairments given by channel copropagation onto the same fiber), as will be specified later on. This assumption allowed us to keep the complexity relatively low, thus allowing us to explore all the features mentioned above.

The rest of this work is organized as follows: Section II summarizes some existing works on this topic; Section III presents our reference model of the translucent node and DWDM system; Section IV summarizes the concept of the connectivity graph, emphasizing its applicability to the IA translucent design, and briefly explains the physical impairment model adopted in our study; Section V describes the IA-ILP formulation, the hybrid ILP/heuristic procedure, and the analytical framework for evaluating the network cost; Section VI shows the results of the planning phase under static traffic for two network topologies; the conclusions are drawn in Section VII.

\section{PRIOR WORK}

Translucent network planning typically aims at employing the smallest possible number of regeneration resources. Various European projects (i.e., DICONET [11], PHOSPHORUS [12], NOBEL [13]) dealt with translucent dimensioning. In other works $[9,14,15]$ a particular emphasis is given on where to deploy regenerators to minimize the number of rejected connections in a dynamic scenario.

Various IA-ILP formulations have been proposed in literature. Some of them deal with the routing with regenerator problem (RRP), where the subset of regenerating nodes is known a priori and lightpaths are routed under physical impairment constraints [15]. Some works [15,16] deal with the regenerator placement problem (RPP), considering a maximum distance, or maximum number of links, that a lightpath may cross without regeneration. In [17] the distance criterion is mixed with a game theoretic approach. The distance criterion can be an effective way to avoid including propagation impairments as constraints in ILP formulations, but its weakness compared with an IA-ILP design strategy has been demonstrated in [18]. An ILP formulation dealing with translucent design in mixed-line-rate (MLR) networks has been proposed in [19].

In $[9,14]$ the connectivity graph (see a formal definition in Section IV) has been proposed to solve RPP alone, and in [16] it is applied jointly with traffic grooming. Effective heuristic methods have been described in [20], though they still do not optimize the number of TXPs and fibers, but only the number of regeneration sites. An IA-ILP formulation [21] that considers amplified spontaneous emission noise and polarization mode dispersion as physical impairments has also been proposed. That work is based on the assumption that regenerating nodes are not allowed to carry out wavelength conversion and that the number of DWDM systems on each link is preassigned. Moreover, the number of TXPs installed in each regenerating node is preassigned: such an assumption is not compatible with a green-field dimensioning phase, where the total number of resources should be a result of planning rather than being fixed a priori.

This study extends our previous work [10] by providing a comprehensive analytical design framework that jointly addresses network resource dimensioning (DWDM systems, TXPs, regenerating nodes) and network cost evaluation in terms of capital expenditure (CAPEX) and operational expenditure (OPEX).

\section{TRANSLUCENT OTN MODEL}

The OTN model that we consider here comprises two classes of elements: translucent optical nodes and unidirectional DWDM systems. A translucent node consists of an all-optical nonblocking switching fabric equipped by a number of $3 \mathrm{R}$ units (TXPs). It is able to switch an optical signal transparently from an input port to an output port (introducing only attenuation) while it can perform regeneration and $\lambda$-conversion on a subset of signals, according to the number of its TXPs. The core of the translucent node is the optical switching fabric. Some of its ports are dedicated to local tributaries via tunable optoelectronic devices. Other ports are used by the transiting signals, which can cross the fabric without regeneration. The rest of its ports are connected to the pool of TXPs (see Fig. 1). A DWDM line system is composed of a fiber, a set of optical line erbium-doped fiber amplifiers (EDFAs), boosters, and preamplifiers together with a wavelength multiplexer (mux) and a demultiplexer (demux) at each terminal of the system. We assume that in our OTN model all optical links are equipped with the same type of DWDM systems; a link can host one or more line systems at the same time. In each DWDM system a preassigned maximum number of wavelength channels can be lit on, and this number is assumed to be the same for all the systems of the network. We do not consider other optical-domain processing devices such as dispersion compensators, WDM channel equalizers, etc. The OTN model in this paper takes only the optical transport layer into account, leaving the higher layers for future extensions. Therefore we assume no optical channel (OCh) or client-layer processing (e.g., traffic grooming) is performed at transit nodes.

\section{Preliminary Concepts for Translucent NETWORK DESIGN}

Operational research has been used to support translucent design in many works. As an example, in [9] the authors 


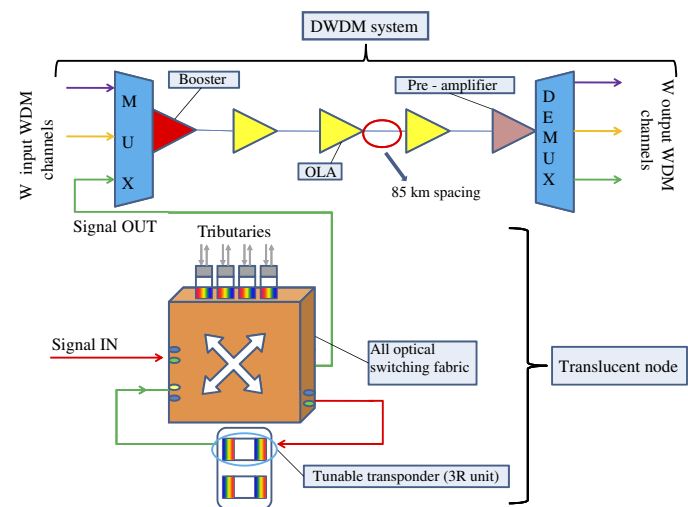

Fig. 1. (Color online) Node model and DWDM system for a translucent network.

addressed the issue of finding the set of regenerating nodes by solving a modified version of the vertex covering problem by an ILP formulation. A heuristic for solving a variation of the hitting set problem has been used by authors in [20]. The concept of the transparency island (TI) has been defined in $[22,23]$ as the set of nodes that can be reached by a node along the shortest path without using $3 \mathrm{Rs}$. In order to connect two nodes that are not in each other's TI, regeneration units are needed at some intermediate nodes. Obviously the higher is the bit error rate (BER) threshold of a lightpath tolerated at the receiver, the wider the TIs become.

\section{A. The k-p Connectivity Graph}

In this work we extend the connectivity graph [9] that is based on the concept of TIs. Given a physical graph of the network $G(N, A)$, where $N$ is the set of nodes and $A$ is the set of directed physical links, the connectivity graph $G^{\prime}\left(N, A^{\prime}\right)$ in its simplest form is obtained by setting $A^{\prime}=\varnothing$, then adding a logical link to $A^{\prime}$ connecting two nodes $(i, j) \in N$, if $j$ belongs to the TI of $i$ and vice versa. It should be noted that $G^{\prime}\left(N, A^{\prime}\right) \supseteq G(N, A)$, unless direct physical links connecting two adjacent nodes are not well dimensioned, i.e., unless the optical signal quality falls below the BER threshold and an in-line regeneration is required. Since we do not aim at placing in-line regenerators, threshold values are chosen according to the two following criteria: a) every link can be traversed without in-line regenerations; b) there is the need for regeneration sites.

In a previous work we enhanced the basic connectivity graph to a "wavelength-aware" connectivity graph; that is, each wavelength or each class of wavelengths with similar transparent reach values has its own connectivity graph [24]. We have shown its effectiveness as a tool for translucent network design.

In this study for each logical edge in $A^{\prime}$, which means for every transparent path that can be established between every pair of source-destination nodes in the network, we identify the mapping of at most $k$ shortest paths over the physical graph. This means that we extend the basic connectivity graph to a $k-p$ connectivity graph in which each $(i, j) \in A^{\prime}$ represents a set composed by at most $k$ physical routes. It may happen that for some logical edges not all of the $k$ paths can exist, because of the network topology or the physical layer impairments. Obviously it depends on the signal quality

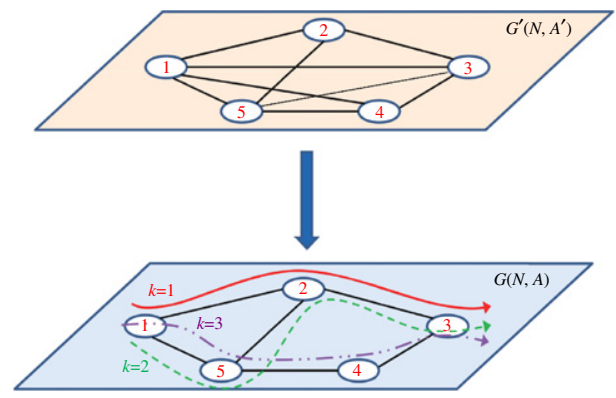

Fig. 2. (Color online) Mapping of the logical edge 1-3 over $k=3$ physical routes.

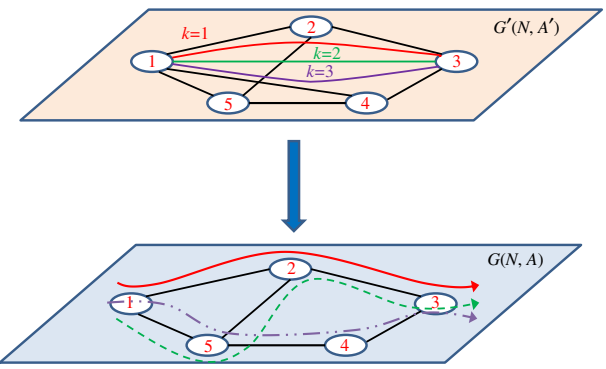

Fig. 3. (Color online) Mapping of each logical edge between 1-3 over one physical route.

threshold considered at the receiver. So we first calculate the $k$ paths by means of Yen's procedure [25], and then we check the path's feasibility under the BER constraint (enforced by our physical model that is briefly presented in Subsection IV.B). The more meshed is the network, the more chances to have all $k$ paths feasible within a reasonable threshold. This will be discussed below in Section VI.

We denote the $k-p$ connectivity graph by $k-p G^{\prime}\left(N, A^{\prime}\right)$. There are two equivalent ways to present the concept of the $k-p$ connectivity graph:

1) The $k-p G^{\prime}\left(N, A^{\prime}\right)$ is a directed graph that has the same number of edges as the simple $k=1 G^{\prime}\left(N, A^{\prime}\right)$, but, when routing connections, each edge in the $k-p G^{\prime}\left(N, A^{\prime}\right)$ represents at most $k$ physical paths instead of just the shortest path $(k=1)$ (see Fig. 2)

2) The $k-p G^{\prime}\left(N, A^{\prime}\right)$ is a directed graph that has at most $k$ edges between each pair of nodes that can be connected without using regenerators. Each edge represents a single path over the physical layer (see Fig. 3).

As an example, in Fig. 2 we represent the mapping of a transparent edge (1-3) over three paths on the physical layer (1-2-3, 1-5-2-3, 1-5-4-3). For the sake of clarity we show the undirected version of the logical graph. In the same way, in Fig. 3 each logical edge connecting node 1 to node 3 is mapped to a different physical path in $G(N, A)$. In the following we adopt the definition related to Fig. 2.

We recall that if there exists an edge in $A^{\prime}$ between two nodes, a request connecting them can be set up along one of the associated $k-p$ 's without intermediate regeneration. The benefit of considering the $k-p$ 's is straightforward. If we had considered $k=1 G^{\prime}\left(N, A^{\prime}\right)$ and there were not free lambdas either on the physical link 1-2 or 2-3, a lightpath between nodes $(1,3)$ would have been set up, requiring at least one 
regenerator site and one TXP instead of none, e.g., on the logical path 1-5-3 or 1-4-3 and two regenerating nodes and two TXPs on the path 1-5-4-3 instead of none. That is because when we route connections by using the connectivity graph a regenerator has to be placed every time a connection has to cross more than one logical link.

\section{B. Model for Physical Layer Impairments}

An important feature when dealing with IA design is the model for the impairments. A model must be used to evaluate whether a path is feasible for a given quality threshold, to create the edges of the connectivity graph. In fact, the connectivity graph itself, after this preprocessing phase, carries this information by its edges. Solving the RPP becomes much easier, since no constraints about the optical signal degradation have to be added to the mathematical model. The only constraint is that a connection can traverse at most one transparent link in $A^{\prime}$ without an intermediate regeneration. Otherwise, a regenerator has to be placed.

Our physical layer impairment model is based on the computation of Personick's $Q$ factor [26]. The $Q$ factor can be computed by different methods, e.g., by pure analysis or by physical layer simulations. Our model adopts a semi-empirical method, as described in [27], which is applicable to WDM systems with on-off keying (OOK)-modulated channels with line rates up to $40 \mathrm{Gbits} / \mathrm{s}$. A signal quality threshold $Q_{t h}$ is used to evaluate whether the optical signal needs regeneration or not. Note that $Q_{t h}=17 \mathrm{~dB}$ roughly corresponds to a BER of $10^{-12}$ (assuming no forward error correction (FEC) performed). We considered the following propagation parameters:

- Average amplification span length, $85 \mathrm{~km}$;

- Fiber loss, $0.23 \mathrm{~dB} / \mathrm{km}$;

- Cable margin, $2 \mathrm{~dB}$;

- Quantum noise, $-58 \mathrm{dBm}$;

- Noise figure of line and preamplifier, $5 \mathrm{~dB}$;

- Noise figure of a booster, $6 \mathrm{~dB}$;

- Power lever at signal launch, $3 \mathrm{dBm}$;

- Optical signal-to-noise ratio degradation for optical node bypass, $2 \mathrm{~dB}$.

An example of the calculation of the $Q$ factor has been shown in [28]. The model used in this paper takes the following impairments into account: amplified spontaneous emission noise, loss, self-phase modulation (SPM), cross-phase modulation (XPM) and four-wave mixing (FWM) nonlinear effects. Cross-phase modulation and four-wave mixing are the so-called "dynamic impairments," as they represent the impairments given by channel copropagation onto the same fiber. They may severely limit the signal's transmission quality, particularly when different data-rate and phase-shift-keying (PSK) modulation formats are considered. In fact, when OOK and PSK modulated signals are both used, the effect of phase noise on PSK-modulated channels becomes no longer negligible [29]. Such noise is brought by the OOK signal amplitude, which remains almost constant in time. As a matter of fact, in single-line rate networks or even in mixed rate networks where intensity-based modulation is used, such effects are not that detrimental [30].

Since our study concerns $10 \mathrm{Gbit} / \mathrm{s}$ signals with OOK modulation, we have addressed dynamic impairments in a less com- plex way than modeling them into our formulation, which can be defined as a worst-case planning scenario. Particularly, we calculate the $Q$ factor of a certain lightpath considering that all $W$ lambdas propagate together for the same source-destination node pair. Apart from a meticulous study of this aspect like that performed by the authors of [31] and [32], our approach still then accounts for such kinds of impairments while keeping the complexity relatively low to allow us to explore other complexity dimensions (i.e., fiber placement, $k$ paths on the connectivity graph, wavelength conversion). This is also the case for crosstalk in the nodes, for which we have assumed a global optical signal-to-noise ratio degradation at each optical bypass. As for any worst-case approach, a certain degree of overestimation has to be accounted for if the hypothesis does not hold.

Polarization mode and chromatic dispersions are considered as totally compensated at the receiver. We further assume that all WDM channels are modulated at $10 \mathrm{Gbit} / \mathrm{s}$. Note that the inclusion of other impairments in the $Q$-factor computation or the adoption of more sophisticated impairment models would not affect the complexity of the problem, since computation would be carried out during the preprocessing phase.

\section{Translucent Design Approaches}

In this section we discuss the proposed IA-ILP formulation exploiting the $k-p$ connectivity graph (from now on we refer to this kind of design approach as $k-p$ IA-ILP). We have developed a framework to include several important features required by network operators [20], such as guaranteeing any-to-any connectivity and supporting protection path and/or, more generally, multiple feasible paths among network nodes. We demonstrate how our model can be easily extended to consider some deployment constraints, such as when some nodes may not be selected as regenerator sites (due to equipment-footprint or power constraints) or when a regenerator site may support only a limited number of regenerators, etc. Then, we provide the algorithmic details of a hybrid model (mixed ILP and heuristic briefly described in [10]) which will be used below as comparison for planning results.

\section{A. The $k-p$ IA-ILP Formulation}

In the following we report the $k-p$ IA-ILP formulation. Let us define all the data, variables and constraints involved.

- Data

- $G(N, A)$ : directed physical graph of the network, where $N$ is the set of nodes and $A$ represents the set of physical links $(l, m)$.

- $k-p G^{\prime}\left(N, A^{\prime}\right)$ : directed $k-p$ connectivity graph of the network, where $N$ is the set of nodes and $A^{\prime}$ is the set of logical edges $(i, j)$.

- $R$ : set of connection requests.

- $s_{r}$ : source node of the connection request $r \in R$.

- $d_{r}$ : destination node of the connection request $r \in R$.

- $W$ : set of wavelengths on each DWDM system.

- $K_{i j}$ : set of the indices $q$ of the physical paths for the logical edge $(i, j) . K_{i j} \subseteq\{1,2, \ldots, k\}$, as not all the $k$ paths can exist for a given $(i, j)$.

- $P_{i j q}$ : set of physical links crossed by the $q_{t h} \in K_{i j}$ path associated with the logical edge $(i, j) \in A^{\prime}$. 
- Variables

- $X_{i j w q}^{r}: X_{i j w q}^{r}=1$ if the traffic demand $r \in R$ crosses the logical edge $(i, j) \in A^{\prime}$ and it is routed over the $q_{t h}$ physical path with wavelength $w \in W ; X_{i j w q}^{r}=0$ otherwise.

- $Y_{i}: Y_{i}=1$ if the node $i \in N$ is a regenerating node; $Y_{i}=0$ otherwise.

- $F_{l m}$ : number of DWDM systems installed on the physical link $(l, m) \in A$.

- Objective function: minimize the number of DWDM systems, regenerator sites and TXPs to route all connection requests,

$$
\operatorname{Min}: \alpha \sum_{(l, m)} F_{l m}+\beta \sum_{i} Y_{i}+\gamma \sum_{r} \sum_{(i, j)} \sum_{w, q} X_{i j w q}^{r} .
$$

\section{- Constraints}

- Solenoidality,

$$
\begin{gathered}
\sum_{w, q, j} X_{i j w q}^{r}-\sum_{w, q, j} X_{j i w q}^{r}=\left\{\begin{aligned}
1 & \text { if } i=s_{r} \\
-1 & \text { if } i=d_{r} \\
0 & \text { otherwise }
\end{aligned}\right. \\
\forall r \in R \wedge \forall i \in N .
\end{gathered}
$$

- Regeneration,

$$
\begin{gathered}
\sum_{w, q, i} X_{i j w q}^{r} \leq Y_{j} \\
\forall r \in R \wedge \forall j \in N \mid j \neq s_{r} \wedge j \neq d_{r} .
\end{gathered}
$$

- Capacity,

$$
\begin{gathered}
\sum_{r}\left[\sum_{(i, j), q \mid(l, m) \in P_{i j q}} X_{i j w q}^{r}\right] \leq F_{l m} \\
\forall(l, m) \in A \wedge \forall w \in W .
\end{gathered}
$$

- Loopless paths,

$$
\begin{aligned}
& \sum_{w}\left[\sum_{(i, j), q, l \mid(l, m) \in P_{i j q}} X_{i j w q}^{r}\right] \leq 1 \\
& \forall r \in R \wedge \forall m \in N \mid m \neq s_{r} \wedge m \neq d_{r} .
\end{aligned}
$$

Let us start by presenting the coefficients in Eq. (1). $\alpha$ and $\beta$ are related by the condition $\alpha+\beta=1$. Given the intrinsically multiobjective nature of this optimization problem, devising an appropriate objective function is a complex task. As we discuss below, we have assigned a larger value to $\alpha$ when minimizing the number of DWDM systems (sparse translucent design approach), while $\beta$ is assigned a larger value when minimizing the number of regeneration sites (clustered translucent design approach). The inclusion of the third term of Eq. (1) (assigning a very small value to $\gamma$ ) allows us to obtain the solution with the smallest number of TXPs, among all the feasible ones. In fact, as we are routing connections on a connectivity graph, each intermediate node of a connection acts as a regeneration point, and one TXP has to be assigned to the lightpath. Then, if we minimize the number of flow variables, we minimize the number of TXPs.

Equation (2) represents the flow conservation constraints that allow routing traffic demands over the $k-p$ connectivity graph. Wavelength conversion has been taken into account by summing over the index $w \in W$ when considering intermediate nodes $\left(j \neq d_{r} \wedge j \neq s_{r}\right)$. With this simple expedient the signal is not forced to keep its wavelength when crossing intermediate nodes in the connectivity graph for a given demand. That is allowed, since the intermediate nodes of a connection routed on a connectivity graph act as a regeneration point. As we show in Section VI, allowing regeneration nodes to change the wavelength is a more realistic and computation time-saving planning scenario. If we had to consider the wavelength continuity constraint, as in [21], we should remove the index $w \in W$ from the summation by rewriting the constraint in Eq. (2) for intermediate nodes as follows:

$$
\begin{gathered}
\sum_{q, j} X_{i j w q}^{r}-\sum_{q, j} X_{j i w q}^{r}=0 \\
\forall w \in W \wedge \forall r \in R \wedge \forall i \mid(i, j) \in A^{\prime} .
\end{gathered}
$$

The RPP can be solved with Eq. (3) by imposing that a node $j$ that is used as intermediate node for some connection will be a regeneration node. So, no preprocessing phase calculating the regenerations segments like that in [18] has to be carried out, and no physical impairments need to be taken directly into account in the formulation.

Equation (4) enforces the capacity constraint and maps the logical edges over the physical links. For each link in $A$, the total number of lightpaths that are routed at a given wavelength $w$ on a logical path related to that physical link $\left((i, j), q \mid(l, m) \in P_{i j q}\right)$ must be equal to or less than the number of DWDM systems installed on that link. In [21] the authors conceived this constraint from a different point of view. Given a logical edge, they constrained the utilization of a given wavelength on each physical link related to that edge. Then they formulated another constraint for the maximum capacity allowed on each physical link. This implicitly does not constrain the routing to employ different wavelengths when crossing two different logical edges that share a physical link. Moreover, our approach allows us to save $\left|A^{\prime}\right|$ constraints.

Equation (5) prevents two transparent segments of a path (i.e., two consecutive logical edges) from sharing the same node or physical link by enforcing that each intermediate node of a connection can support at most one incoming traffic flow for a given demand. This is a common problem when using auxiliary graphs for network design and this constraint becomes more essential in our study, since we deal with a $k-p$ connectivity graph where for each logical edge at most $k$ physical shortest paths are accounted for.

Therefore our formulation allows us to completely design a translucent network by placing DWDM systems, selecting regeneration sites, and placing TXPs to satisfy a given static traffic. The RPP and the routing, fiber and wavelength assignment with regenerator problem (RFWA-RP) are carried out on the $k-p$ connectivity graph, allocating resources by mapping each logical edge over at most $k$ paths on the physical layer. Moreover, the wavelength conversion capability of TXPs has been taken into account.

As we mentioned before, this model can be easily upgraded to encompass some other useful features [20].

1) Setting regeneration sites: Choosing regenerator sites by giving priority to some nodes instead of to others can be 
easily accomplished by associating a certain cost with each node. Such a cost vector $C_{i}$ would multiply $Y_{i}$ in Eq. (1).

2) Constraining the maximum number of transponders: Some regenerating nodes may not to be allowed to host many TXPs due to space limitation, heat dissipation, etc. This constraint can be modeled as follows:

$$
\sum_{w, q, i}\left[\sum_{r \mid s_{r}, d_{r} \neq j} X_{i j w q}^{r}\right] \leq M_{j} \forall j \in N .
$$

In Eq. (7), for each intermediate node $j$ the total number of incoming flows in $k-p G^{\prime}\left(N, A^{\prime}\right)$ (number of TXPs) must be equal to or less than a given value $\left(M_{j}\right)$ related to that node.

3) Protection: We also provide here equations to extend our model to the dedicated path-protection case, preventing link failures. Since we have already introduced an index $r$ that differentiates each single connection request between a given source-destination pair, the main flow variable $X_{i j w q}^{r}$ does not change.

For each request, two lightpaths have to be routed. Equation (2) becomes

$$
\begin{gathered}
\sum_{w, q, j} X_{i j w q}^{r}-\sum_{w, q, j} X_{j i w q}^{r}=\left\{\begin{aligned}
2 & \text { if } j=s_{r} \\
-2 & \text { if } j=d_{r} \\
0 & \text { otherwise }
\end{aligned}\right. \\
\forall r \in R \wedge \forall i \mid(i, j) \in A^{\prime} .
\end{gathered}
$$

Equation (9) remains pretty much the same as Eq. (3), just taking into account that the demands are doubled; so for each intermediate node of a given connection the total incoming flow is constrained to the availability of regeneration devices:

$$
\begin{gathered}
\sum_{w, q, i} X_{i j w q}^{r} \leq 2 \cdot Y_{j} \\
\forall r \in R \wedge \forall j \in N \mid j \neq s_{r} \wedge j \neq d_{r} .
\end{gathered}
$$

Equation (9) allows working and protection paths to share the same regenerating node, whereas using Eq. (3) instead would forbid that. This may increase the number of required regeneration nodes, but it may be worth that compromise to improve the quality of the dedicated protection, which in turn depends on operators' needs. However, in our case study we have used Eq. (9), since the scope was to route the protection path to prevent link failures.

The constraint that enforces the link disjointness of the working and the protection paths is expressed by Eq. (10). A given physical link $(l, m)$ can support at most one single traffic flow of a given demand $r$ (working or protection). Moreover, if the upstream direction is used for either working or protection, the downstream is forbidden and vice versa. Thus, only one flow variable per connection $r$ can be set to one on that link $(l, m)$ independently of the number of fibers and the chosen wavelength $w$ :

$$
\begin{gathered}
\sum_{w} \sum_{(i, j), q \mid(l, m) \in P_{i j q}}\left(X_{i j w q}^{r}+X_{j i w q}^{r}\right) \leq 1 \\
\forall(l, m) \in A \wedge \forall r \in R .
\end{gathered}
$$

Although connections are actually routed over the $k-p$ connectivity graph [according to Eq. (8)], it should be noted that the link disjointness [Eq. (10)] has to be directly applied to the physical graph, while it cannot be imposed on the connectivity graph because two different logical edges can share some physical links. Moreover, even a single logical edge could also be traversed by the working and protection lightpath of a certain demand at the same time, since it may happen that two $q_{t h}$ physical paths associated with the same logical edge are link disjoint. Equations (8) and (10) prevent this case.

\section{B. The Hybrid Method}

In order to decrease computational time we split the problem of translucent design into two steps as follows:

1) Selection of the regenerating nodes (RPP),

2) Dimensioning of resources (RFWA-RP).

The clustered design strategy is carried out first by finding the regenerator locations via a simple IA-ILP and then by proceeding to the second step with TXP locations constrained for the routing. The sparse strategy has been accomplished by selecting each node as regenerating and then by proceeding to the next step. This method was first presented in our previous publication [10]. For brevity, in the following we give the details that were not reported in [10].

The RFWA-RP algorithm is based on a metric to compute the cost of a given path. For an $H$-hop path $P$ routed on links having length $L_{i, P}$, the cost is given by

$$
C_{P}=\sum_{i=1}^{H} L_{i, P}+R_{P} \cdot C_{\mathrm{reg}} .
$$

The number of TXPs along the path $P$ needed to satisfy the signal quality constraint is represented by $R_{P}$ associated with a certain cost $C_{\text {reg. }}$. The main steps of the hybrid heuristic algorithm are summarized in Algorithm 1. Due to the

\section{Algorithm 1 Hybrid method}

\section{FIRST STEP:}

- Input: connectivity graph $G^{\prime}\left(N, A^{\prime}\right)$, traffic matrix;

1) IA-ILP formulation;

- Output: regenerators location; SECOND STEP:

- Input: physical layer graph $G(N, A)$, maximum number of WDM channels W per DWDM system, regenerator locations, impairment model, static traffic matrix;

1) The network is equipped with one TXP per selected regenerating node and one DWDM system per link;

2) Process the first demand minimizing $C_{P}$. Wavelength assignment is accomplished by the first-fit criterion;

3) Nodes with no free TXPs are provided with one extra TXP. Each link with no free wavelengths on the already installed DWDM systems is provided with one additional DWDM system;

4) Repeat steps 3 and 4 until all connections have been set up;

5) Unused TXPs and DWDM systems, if any, are removed;

- Output: number of TXPs and DWDM systems. 
nonmonotonic behavior of the $Q$ factor, the minimum-cost-path searching algorithm cannot be as simple as the classical version of Dijkstra. In our situation candidate paths cannot be immediately discarded on the basis of their costs: a more expensive subpath may become the best solution if the $Q$ factor of the minimum-cost subpath falls below the threshold $Q_{t h}$. This has a deep consequence, since the computational complexity tends to grow exponentially as the network is explored starting from the source node. The concept of Pareto dominance can be adapted to this case: given an $H$-hop path $P_{1}$ and a $K$-hop path $P_{2}$, we say that $P_{1}$ dominates $P_{2}$ if and only if all the following constraints are satisfied:

- $C_{P 1} \leq C_{P 2}$,

- $H \leq K$,

- $Q_{P 1} \leq Q_{P 2}$,

and if at least one constraint holds as strict inequality.

The set of candidate paths is restricted to dominating paths; i.e., each time a path of the set is dominated, it is discarded. In conclusion, our RFWA-RP algorithm is a $Q$ and regenerator-location-constrained breadth-first-search over nondominated paths.

\section{Analytical Comparison of Translucent Design Strate- gies}

As we mentioned above, there are two main approaches for translucent network design [10]: a) clustered translucent or b) sparse translucent. The latter allows us to decrease the quantity of DWDM systems, at the cost of increasing the number of regenerating nodes and TXPs; the former allows us to reduce the number of regenerating sites and TXPs, but it increases the number of DWDM systems. Our $k-p$ IA-ILP approach allows us to switch from one strategy to another by changing the values of coefficients $\alpha$ and $\beta$ in the objective function.

In the sparse method connections are routed along longer paths than in the clustered one. This is due to the need to exploit the free capacity in each link to minimize the number of DWDM systems. In the clustered approach, minimizing regeneration locations also implies fewer chances to undergo wavelength conversion. Therefore clustering TXPs in a subset of nodes is economically effective only when fully transparent network nodes have a significantly lower cost compared with nodes hosting $3 \mathrm{R}$ units and thus compensate for the extra CAPEX for more DWDM systems. This scenario can become realistic when the OPEX due to regenerator hosting is high (e.g., larger area occupation in node-housing infrastructures, much larger energy consumptions, need for special equipment for heat dissipation, maintenance costs, etc.).

To relate the OPEX of hosting regenerator devices to the cost of one TXP, we made the following assumptions. Let us consider the network model in Fig. 1 and the cost model presented in $[7]^{1}$ :

- The cost of one ultralong-haul tunable TXP (10 Gbit/s) is equal to that of an optical amplifier (booster, line, and preamplifier).

- The cost of Mux+Demux devices is equal to the cost of one TXP.

${ }^{1}$ We consider a regeneration device (3R unit) made of one TXP (see Fig. 1) while in [7] the authors considered a 3R unit made of two TXPs, though maintaining the same cost relation.
- The total cost of a translucent network is given by the summation of three parameters multiplied by a certain cost: number of regenerating nodes, number of DWDM systems, and number of TXPs.

- The cost of the optical switching fabric in Fig. 1 has been neglected, since it is installed at every node.

Given our assumptions, we can model the overall network cost $Y$ by a linear function:

$$
Y=C_{3 R} \cdot N_{\text {nodes }}+C_{\text {syst }} \cdot N_{\text {syst }}+C_{\text {txp }} \cdot N_{\text {txp }},
$$

where $C_{3 R}$ is the OPEX of a regenerating node, $C_{\text {syst }}$ is the cost of a DWDM system and $C_{\text {txp }}$ is the cost of one tunable TXP, and they represent the CAPEX. $C_{\text {syst }}$ varies with link lengths by considering the number of amplifiers that compose the DWDM system. The $N_{\text {nodes }}, N_{\text {syst }}$, and $N_{\text {txp }}$ refer respectively to the number of regenerating nodes, DWDM systems, and TXPs as output of the planning phase. If each cost is normalized to the cost of one TXP, we obtain

$$
Y^{\prime}=\frac{C_{3 R}}{C_{\mathrm{txp}}} \cdot N_{\text {nodes }}+\frac{C_{\mathrm{syst}} \cdot N_{\mathrm{syst}}}{C_{\mathrm{txp}}}+N_{\mathrm{txp}} .
$$

$Y^{\prime}$ is the normalized network cost, and Eq. (12) represents a line as a function of the variable $C_{3 R} / C_{\text {txp }}$ (normalized OPEX with respect to the cost of one TXP). The $y$ intercept of this line represents the total normalized cost of devices, while $N_{\text {nodes }}$ is the slope of the linear function.

It is also worth mentioning that the cost functions in Eq. (1) and in Eq. (12) are different. The former is the objective function of our optimization engine and the coefficients $\alpha, \beta$ and $\gamma$ are used to switch from the clustered to the sparse design strategy. The latter, instead, is used to evaluate whether one strategy is more cost-effective than the other as a function of the number of resources obtained after the design session (CAPEX) and on the basis of the normalized OPEX.

\section{ILlustrative NumericAl RESUlts}

Our design experiments have been performed using the NSFNET and COST239 networks (see Fig. 4). NSFNET has 14 nodes and 22 bidirectional links, the maximum nodal degree is 4 , and the average link length is $1936 \mathrm{~km}$. COST239 has 11 nodes and 26 bidirectional links, the maximum nodal degree is 6 , and the average link length is $577 \mathrm{~km}$. For both networks, we have used a uniform static matrix of demands including one bidirectional request between each pair of nodes. All the demands have been considered at $10 \mathrm{Gbit} / \mathrm{s}$, and the maximum capacity $W$ of all DWDM systems is set to $4,8,16$, and 40
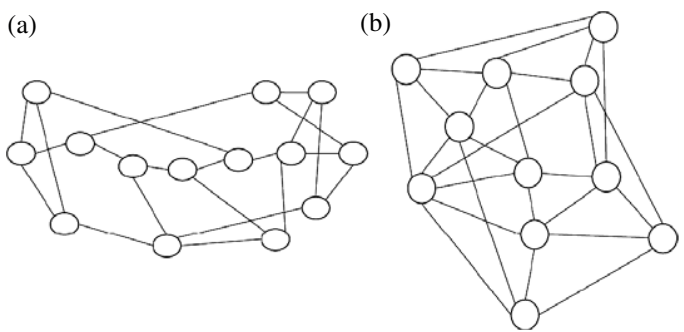

Fig. 4. (a) NSFNET; (b) COST239. 
wavelengths. We have used CPLEX 12.0 on a workstation equipped with $8 \times 2.00 \mathrm{GHz}$ processors and with 32 Gbytes of RAM. The optical signal quality threshold for the NSFNET has been set to $Q_{t h}=13 \mathrm{~dB}$, which roughly corresponds to a maximum transparent reach of $4800 \mathrm{~km}$. As for the COST239, the threshold has been set to $Q_{t h}=21 \mathrm{~dB}$, which roughly corresponds to a transparent reach of $1450 \mathrm{~km}$. For both networks, $Q_{t h}$ values are chosen on the basis of their longest link; thus at least any link can be traversed.

\section{A. The Advantage of the $k-p$ Connectivity Graph}

The comparison between the clustered versus the sparse dimensioning approach in terms of total number of regenerating nodes, installed TXPs (TXPs, here acting also as $\lambda$-converters), and unidirectional DWDM systems for the two networks is shown in Table I. Results of the same network obtained by the opaque implementation and hybrid method have been also reported. We have conceived the opaque strategy by routing in a greedy way all connections along the shortest path and by adding one TXP at each transit node per wavelength on the incoming/outgoing DWDM systems. As for the opaque and the hybrid, wavelength assignment is accomplished by the first-fit criterion. We have used $k=1,2,3$ for NSFNET and $k=1,2,3,4,5$ for COST239. Table I also reports the relative difference with respect to the best solution (i.e., corresponding to $k=3$ and to $k=5$ for NSFNET and COST239, respectively) for each value of TXPs, DWDM systems, and regenerating

TABLE I

Network-Planning Results With Static TraffiC

\begin{tabular}{|c|c|c|c|}
\hline Design method & $\begin{array}{l}\text { No. of reg. } \\
\text { nodes }\end{array}$ & No. of TXPs & $\begin{array}{l}\text { No. of unidir. } \\
\text { DWDM systems }\end{array}$ \\
\hline \multicolumn{4}{|c|}{ NSFNET, sparse translucent, $W=16$, uniform traffic } \\
\hline $\begin{array}{l}k-p \text { IA-ILP } k=1 \\
k-p \text { IA-ILP } k=2 \\
k-p \text { IA-ILP } k=3 \\
\text { HYBRID } \\
\text { OPAQUE }\end{array}$ & $\begin{array}{lc}9 & (+50 \%) \\
7 & (+17 \%) \\
6 & / / \\
8 & (+33 \%) \\
14 & / /\end{array}$ & $\begin{array}{ll}98 & (+31 \%) \\
78 & (+4 \%) \\
75 & / / \\
50 & (-33 \%) \\
688 & / /\end{array}$ & $\begin{array}{lc}32 & (+0 \%) \\
32 & (+0 \%) \\
32 & / / \\
42 & (+31 \%) \\
42 & / /\end{array}$ \\
\hline \multicolumn{4}{|c|}{ NSFNET, clustered translucent, $W=16$, uniform traffic } \\
\hline $\begin{array}{l}k-p \text { IA-ILP } k=1 \\
k-p \text { IA-ILP } k=2 \\
k-p \text { IA-ILP } k=3 \\
\text { HYBRID } \\
\text { OPAQUE }\end{array}$ & $\begin{array}{lc}1 & (+0 \%) \\
1 & (+0 \%) \\
1 & / / \\
1 & (+0 \%) \\
14 & / /\end{array}$ & $\begin{array}{lc}48 & (+9 \%) \\
46 & (+5 \%) \\
44 & / / \\
46 & (+5 \%) \\
688 & / /\end{array}$ & $\begin{array}{ll}52 & (+10 \%) \\
48 & (+2 \%) \\
47 & / / \\
54 & (+15 \%) \\
42 & / /\end{array}$ \\
\hline \multicolumn{4}{|c|}{ NSFNET, clustered translucent, $W=40$, uniform traffic } \\
\hline $\begin{array}{l}k-p \text { IA-ILP } k=1 \\
k-p \text { IA-ILP } k=2 \\
k-p \text { IA-ILP } k=3\end{array}$ & $\begin{array}{ll}2 & (+100 \%) \\
1 & (+0 \%) \\
1 & / /\end{array}$ & $\begin{array}{ll}50 & (+19 \%) \\
44 & (+5 \%) \\
42 & / /\end{array}$ & $\begin{array}{ll}40 & (+11 \%) \\
38 & (+5 \%) \\
36 & / /\end{array}$ \\
\hline \multicolumn{4}{|c|}{ COST239, sparse translucent, $W=16$, uniform traffic } \\
\hline $\begin{array}{l}k-p \text { IA-ILP } k=1 \\
k-p \text { IA-ILP } k=2 \\
k-p \text { IA-ILP } k=3 \\
k-p \text { IA-ILP } k=4 \\
k-p \text { IA-ILP } k=5 \\
\text { HYBRID } \\
\text { OPAQUE }\end{array}$ & $\begin{array}{ll}9 & (+28 \%) \\
8 & (+14 \%) \\
7 & (+0 \%) \\
7 & (+0 \%) \\
7 & / / \\
3 & (-57 \%) \\
11 & / /\end{array}$ & $\begin{array}{ll}125 & (+212 \%) \\
86 & (+115 \%) \\
46 & (+15 \%) \\
44 & (+10 \%) \\
40 & / / \\
6 & (-85 \%) \\
768 & / /\end{array}$ & $\begin{array}{ll}19 & (+12 \%) \\
18 & (+6 \%) \\
17 & (+0 \%) \\
17 & (+0 \%) \\
17 & / / \\
48 & (+182 \%) \\
48 & / /\end{array}$ \\
\hline \multicolumn{4}{|c|}{ COST239, clustered translucent, $W=16$, uniform traffic } \\
\hline $\begin{array}{l}k-p \text { IA-ILP } k=1 \\
k-p \text { IA-ILP } k=2 \\
k-p \text { IA-ILP } k=3 \\
k-p \text { IA-ILP } k=4 \\
k-p \text { IA-ILP } k=5 \\
\text { HYBRID } \\
\text { OPAQUE }\end{array}$ & $\begin{array}{lc}1 & (+0 \%) \\
1 & (+0 \%) \\
1 & (+0 \%) \\
1 & (+0 \%) \\
1 & / / \\
1 & (+0 \%) \\
11 & / /\end{array}$ & $\begin{array}{ll}5 & (+25 \%) \\
4 & (+0 \%) \\
4 & (+0 \%) \\
4 & (+0 \%) \\
4 & / / \\
6 & (+50 \%) \\
768 \quad / /\end{array}$ & $\begin{array}{ll}46 & (+64 \%) \\
33 & (+18 \%) \\
30 & (+7 \%) \\
28 & (+0 \%) \\
28 & / / \\
48 & (+71 \%) \\
48 & / /\end{array}$ \\
\hline \multicolumn{4}{|c|}{ COST239, clustered translucent, $W=40$, uniform traffic } \\
\hline $\begin{array}{l}k-p \text { IA-ILP } k=1 \\
k-p \text { IA-ILP } k=2 \\
k-p \text { IA-ILP } k=3 \\
k-p \text { IA-ILP } k=4 \\
k-p \text { IA-ILP } k=5\end{array}$ & $\begin{array}{lc}1 & (+0 \%) \\
1 & (+0 \%) \\
1 & (+0 \%) \\
1 & (+0 \%) \\
1 & / /\end{array}$ & $\begin{array}{ll}5 & (+25 \%) \\
4 & (+0 \%) \\
4 & (+0 \%) \\
4 & (+0 \%) \\
4 & / /\end{array}$ & $\begin{array}{ll}34 & (+70 \%) \\
26 & (+30 \%) \\
22 & (+10 \%) \\
20 & (+0 \%) \\
20 & / /\end{array}$ \\
\hline
\end{tabular}

nodes for each translucent design technique so to give a quick insight into how resources vary with $k$. Because of the signal degradation, it may happen that for large networks (e.g., NSFNET) there are no source-destination couples that can be connected by $k=4$ different physical paths. Obviously, in a much more meshed network (e.g., COST239) there are many couples that can be connected by up to $k=5$ physical routes.

As expected, the translucent strategy achieves a clear CAPEX saving compared with the opaque approach. This is due to a better distribution of load in the network compared with the opaque case, in which routing has been implemented as purely shortest path based.

The advantages of the $k-p$ IA-ILP formulation are manifold. Table I shows that there is a significant benefit for using $k>1$ in terms of needed TXPs, particularly in the sparse approach. In fact, the routing algorithm has more chances to find a transparent path, besides the shortest one, to avoid placing useless regenerators. This is much more evident in COST239, since there are more paths a connection can be routed on. Particularly, for the sparse approach, connections are routed over longer paths trying to exploit the free capacity in each link. In this case, the number of DWDM systems cannot decrease so much as TXPs when increasing $k$, since different $k$ shortest paths between a given $(i, j)$ on the logical graph most likely share many physical links.

As for the realistic scenario with $W=40$ wavelengths, we have carried out simulations for both the COST239 and NSFNET networks, but only for the clustered strategy, because for the sparse strategy increasing the number of wavelengths is highly time consuming as shown in Fig. 5. We have shown that also in this case increasing the connectivity degree of the graph leads to some benefits as in the other scenarios.

\section{B. The Hybrid Method}

The hybrid method performs better in the clustered approach than in the sparse one. This is due mainly to the greedy routing and the first-fit wavelength assignment, which do not allow us to exploit the free capacity of the installed DWDM systems. The results obtained by this procedure are close to those achieved by the IA-ILP with $k=1$. Obviously, the hybrid procedure allows us to substantially decrease the computational time complexity of the design procedure: it usually takes some seconds instead of the hours needed by the $k-p$ IA-ILP as shown below.

The same considerations reported in these two subsections apply for the $W=4,8$ wavelengths cases, whose results are not reported.

\section{The Benefit of Wavelength Conversion}

We noticed that allowing conversion instead of imposing the wavelength continuity constraint does not imply a noticeable CAPEX saving, since the two strategies achieved almost the same results. The benefit of wavelength conversion is indeed the computational time needed to converge to the optimal solution. In Fig. 5 we report the computational time vs. the number of wavelengths per fiber for the $k-p$ IA-ILP $(k=1)$ design strategy of the NSFNET with uniform traffic. Our simulations with $k>1$ took on average from some minutes (clustered approach) to tens of hours (sparse approach) to converge to the optimal solution. 


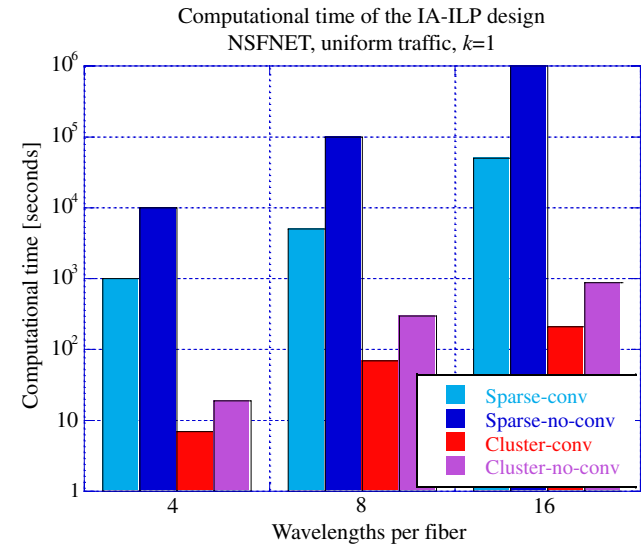

Fig. 5. (Color online) Benefits of wavelength conversion in terms of computational time.

\section{The Network Cost Function Analysis}

The larger is $k$ in the $k-p$ IA-ILP, the more accurate the planning becomes. So we have carried out the calculation of the total cost of the network [Eq. (12)] for NSFNET and COST239 with $k=3$ and $k=5$, respectively. For the cost function analysis, we have considered $W=16$ and wavelength conversion, since we have not carried out the sparse design strategy with $W=40$ as explained above. For each network we have calculated a threshold of OPEX as the value for which the clustered and sparse approaches have the same network cost [point of intersection of the lines representing the two design approaches; see Eq. (12)]. If the total OPEX of hosting regenerators $\left(C_{3 R}\right)$ is greater than this threshold, then the clustered translucent strategy becomes the most cost-effective solution. The threshold values are quite different for NSFNET and COST239. For the latter, the resulting threshold is about 16 times the cost of a single TXP, whereas for the former it is about 27 times the cost of one TXP. These values can be very realistic if we consider that TXPs are hosted in racks and shelves as shown in [33]. To gain insight, let us assume that power consumption is the only contribution to OPEX and that a $10 \mathrm{Gbit} / \mathrm{s}$ TXP consumes $50 \mathrm{~W}$ [33]; then the OPEX threshold is $800 \mathrm{~W}$ for the COST239 and $1350 \mathrm{~W}$ for the NSFNET. These values represent the "cost" threshold of housing racks and shelves to host TXPs in a node for which the clustered strategy outperforms the sparse one.

For small and mesh networks the clustered approach can be cost-effective, since an important part of the cost of a DWDM system is related to its length (according to the assumptions made before). In contrast, for large networks it can be appropriate to aim at minimizing the number of DWDM systems, since long links have a huge cost. We carried out simulations for different values of quality thresholds. Decreasing this value allows the TIs to be increased, and clustered and sparse approaches tend to converge. When increasing the quality threshold (i.e., TIs become smaller and many regenerating nodes are needed), the clustered approach already shows a lower overall network cost for small values of $C_{3 R}$, since the number of regenerating nodes [the slope of the function in Eq. (13)], which is normally high in the sparse strategy, plays a dominant role.

\section{E. Dedicated Path Protection}

In Table II we show the results of the COST239 network for $W=4$ for both the protected and the unprotected scenarios.
TABLE II

Network-Planning Results With Static Traffic for THE Protected and UnProtected Scenarios

\begin{tabular}{|c|c|c|c|}
\hline Design method & $\begin{array}{l}\text { No. of reg. } \\
\text { nodes }\end{array}$ & No. of TXPs & $\begin{array}{l}\text { No. of unidir. } \\
\text { DWDM systems }\end{array}$ \\
\hline \multicolumn{4}{|c|}{ COST239, sparse translucent, $W=4$, uniform traffic, unprotected } \\
\hline $\begin{array}{l}k-p \text { IA-ILP } k=1 \\
k-p \text { IA-ILP } k=2 \\
k-p \text { IA-ILP } k=3 \\
k-p \text { IA-ILP } k=4 \\
k-p \text { IA-ILP } k=5\end{array}$ & $\begin{array}{ll}8 & (+33 \%) \\
7 & (+17 \%) \\
6 & (+0 \%) \\
6 & (+0 \%) \\
6 & / /\end{array}$ & $\begin{array}{ll}31 & (+94 \%) \\
24 & (+50 \%) \\
20 & (+25 \%) \\
18 & (+12 \%) \\
16 & / /\end{array}$ & $\begin{array}{lc}49 & (+9 \%) \\
46 & (+2 \%) \\
45 & (+0 \%) \\
45 & (+0 \%) \\
45 & / /\end{array}$ \\
\hline \multicolumn{4}{|c|}{ COST239, sparse translucent, $W=4$, uniform traffic, protected } \\
\hline $\begin{array}{l}k-p \text { IA-ILP } k=1 \\
k-p \text { IA-ILP } k=2 \\
k-p \text { IA-ILP } k=3 \\
k-p \text { IA-ILP } k=4 \\
k-p \text { IA-ILP } k=5\end{array}$ & $\begin{array}{ll}10 & (+11 \%) \\
10 & (+11 \%) \\
10 & (+11 \%) \\
9 & (+0 \%) \\
9 & / /\end{array}$ & $\begin{array}{ll}132 & (+120 \%) \\
91 & (+52 \%) \\
82 & (+37 \%) \\
67 & (+12 \%) \\
60 & / /\end{array}$ & $\begin{array}{ll}115 & (+10 \%) \\
106 & (+1 \%) \\
105 & (+0 \%) \\
105 & (+0 \%) \\
105 & / /\end{array}$ \\
\hline \multicolumn{4}{|c|}{ COST239, clustered translucent, $W=4$, uniform traffic, unprotected } \\
\hline $\begin{array}{l}k-p \text { IA-ILP } k=1 \\
k-p \text { IA-ILP } k=2 \\
k-p \text { IA-ILP } k=3 \\
k-p \text { IA-ILP } k=4 \\
k-p \text { IA-ILP } k=5\end{array}$ & $\begin{array}{cc}1 & (+0 \%) \\
1 & (+0 \%) \\
1 & (+0 \%) \\
1 & (+0 \%) \\
1 & / /\end{array}$ & $\begin{array}{ll}7 & (+75 \%) \\
4 & (+0 \%) \\
4 & (+0 \%) \\
4 & (+0 \%) \\
4 & / /\end{array}$ & $\begin{array}{ll}65 & (+18 \%) \\
58 & (+5 \%) \\
57 & (+4 \%) \\
55 & (+0 \%) \\
55 & / /\end{array}$ \\
\hline \multicolumn{4}{|c|}{ COST239, clustered translucent, $W=4$, uniform traffic, protected } \\
\hline $\begin{array}{l}k-p \text { IA-ILP } k=1 \\
k-p \text { IA-ILP } k=2 \\
k-p \text { IA-ILP } k=3 \\
k-p \text { IA-ILP } k=4 \\
k-p \text { IA-ILP } k=5\end{array}$ & $\begin{array}{ll}3 & (+50 \%) \\
2 & (+0 \%) \\
2 & (+0 \%) \\
2 & (+0 \%) \\
2 & / /\end{array}$ & $\begin{array}{ll}116 & (+262 \%) \\
42 & (+31 \%) \\
40 & (+25 \%) \\
32 & (+0 \%) \\
32 & / /\end{array}$ & $\begin{array}{ll}142 & (+20 \%) \\
128 & (+8 \%) \\
123 & (+4 \%) \\
120 & (+2 \%) \\
118 & / /\end{array}$ \\
\hline
\end{tabular}

The same considerations as those reported above for the benefit of the $k-p$ 's apply here. As usual, the higher the value of $k$, the more accurate the design results in terms of resources. As for $k=5$, the number of TXPs in the protected case for the sparse and the clustered approaches is, respectively, more than 4 times and equal to 8 times the number in the unprotected case. The number of regenerating nodes slightly increases, but the number of DWDM systems is more than doubled in the two approaches.

\section{CONCLUSIONS}

A global planning procedure for translucent OTNs should be able to choose regenerator sites, place TXPs, and assign DWDM systems to links to satisfy a given static traffic matrix. The connectivity graph is an effective tool to satisfy these requirements, as it reduces the computational complexity of IA-ILP formulations, since all impairments are taken into account in the preprocessing phase. In this work we have enhanced this mathematical tool by introducing the concept of the $k-p$ connectivity graph. The case studies we have reported show the benefit of letting the routing algorithm select among multiple physical paths when solving the RPP and the RFWA-RP, for both the protected and unprotected cases on a logical graph. The presented $k-p$ IA-ILP is flexible: it provides a planning tool to address both the clustered and the sparse strategies, it takes $\lambda$-conversion into account (of which we have reported the benefits in terms of computational time), and it can encompass dedicated path protection and other features useful for a network operator. The hybrid method allows the computational time to be decreased, and its performance in terms of number of resources are closer to the ones achieved by the $k-p$ IA-ILP with $k=1$, particularly when dealing with the clustered implementation.

We have also proposed an analytical framework to evaluate which is the most cost-effective translucent design strategy to be adopted by a network operator. From our simulation results, the clustered translucent approach seems to lead to the least-cost solution when dealing with small mesh networks 
and when the quality threshold required at the receiver is high. This last conclusion will be supported by follow-up research.

\section{ACKNOWLEDGMENTS}

The research leading to these results has received funding from the European Community's Seventh Framework Programme FP7/2007-2013 under grant agreement 247674 (STRONGEST project).

\section{REFERENCES}

[1] S. Gringeri, B. Basch, V. Shukla, R. Egorov, and T. J. Xia, "Flexible architectures for optical transport nodes and networks," IEEE Commun. Mag., vol. 48, pp. 40-50, July 2010.

[2] S. Tibuleac and M. Filer, "Transmission impairments in DWDM networks with reconfigurable optical add-drop multiplexers," $J$. Lightwave Technol., vol. 28, pp. 557-598, Feb. 2010.

[3] B. Ramamurthy, H. Feng, D. Datta, J. P. Heritage, and B. Mukherjee, "Transparent vs. opaque vs. translucent wavelength-routed optical networks," in Tech. Dig. Optical Fiber Communication Conf., 1999, and Int. Conf. on Integrated Optics and Optical Fiber Communication. OFC/IOOC '99, San Diego, CA, USA, 1999, vol. 1, pp. 59-61.

[4] X. Yang and B. Ramamurthy, "Sparse regeneration in a translucent WDM optical network," Proc. SPIE, vol. 4585, pp. $61-70$, 2001.

[5] G. Shen and R. S. Tucker, "Translucent optical networks: the way forward," IEEE Commun. Mag., vol. 45, pp. 48-54, Feb. 2007.

[6] B. Ramamurthy, S. Yaragorla, and X. Yang, "Translucent optical WDM networks for the next-generation backbone networks," in IEEE Global Telecommunications Conf., 2001. GLOBECOM '01, San Antonio, TX, USA, 2001, vol. 1, pp. 60-64.

[7] A. Morea and J. Poirrier, "A critical analysis of the possible cost savings of translucent networks," in Proc. 5th Int. Workshop on Design of Reliable Communication Networks, 2005. (DRCN 2005), Island of Ischia, Italy, 2005, pp. 311-317.

[8] L. Ceuppens, L. Sardella, and A. Kharitonov, "Power saving strategies and technologies in network equipment opportunities and challenges, risk and rewards," in Int. Symp. Applications and the Internet, 2008. SAINT 2008, Turku, 2008, pp. 381-384.

[9] M. S. Savasini, P. Monti, M. Tacca, A. Fumagalli, and H. Waldman, "Regenerator placement with guaranteed connectivity in optical networks," in ONDM'07 Proc. 11th Int. IFIP TC6 Conf. Optical Network Design and Modeling, Athens, Greece, 2007, pp. 438-447.

[10] G. Rizzelli, G. Maier, and A. Pattavina, "Translucent optical network design: a novel two-step method," in 2010 36th European Conf. and Exhibition on Optical Communication (ECOC), Torino, Italy, 2010, pp. 1-3.

[11] "DICONET," http://www.diconet.eu/.

[12] "PHOSPHORUS," http://www.ist-phosphorus.eu/.

[13] "NOBEL," http://www.ist-nobel.org/.

[14] M. S. Savasini, P. Monti, M. Tacca, A. Fumagalli, and H. Waldman, "Trading network management complexity for blocking probability when placing optical regenerators," in Int. Conf. High Performance Switching and Routing, 2008. HSPR 2008, Shanghai, China, 2008, pp. 291-296.

[15] X. Yang and B. Ramamurthy, "Sparse regeneration in translucent wavelength-routed optical networks: Architecture, network design and wavelength routing," Photonic Network Commun., vol. 10, pp. 39-53, July 2005.

[16] A. N. Patel, C. Gao, J. P. Jue, X. Wang, Q. Zhang, P. Palacharla, and T. Naitoet, "Traffic grooming and regenerator placement in impairment-aware optical WDM network," in 2010 14th Conf. Optical Network Design and Modeling (ONDM), Kyoto, Japan, 2010, pp. 1-6.

[17] D. Lucerna, N. Gatti, G. Maier, and A. Pattavina, "On the efficiency of a game theoretic approach to sparse regenerator placement in WDM networks," in IEEE Global Telecommunica- tions Conf., 2009. GLOBECOM 2009, Honolulu, HI, USA, 2009, pp. $1-6$.

[18] K. Katrinis, A. Tzanakaki, and G. Markidis, "Impairment-aware WDM network dimensioning with optimized regenerator placement," in Nat. Fiber Optic Engineers Conf., San Diego, CA, USA, 2009, NThE4.

[19] A. Nag and M. Tornatore, "Transparent vs. translucent optical network design with mixed line rates," in Optical Fiber Communication Conf., San Diego, CA, USA, 2009, OWI7.

[20] C. V. Saradhi, A. Zanardi, R. Fedrizzi, E. Salvadori, G. M. Galimberti, A. Tanzi, G. Martinelli, and O. Gerstel, "A framework for regenerator site selection based on multiple paths," in Optical Fiber Communication Conf., San Diego, CA, USA, 2010, OTuG7.

[21] W. Zhang, J. Tang, K. Nygard, and C. Wang, "REPARE: Regenerator placement and routing establishment in translucent networks," in IEEE Global Telecommunications Conf., 2009. GLOBECOM 2009, Honolulu, HI, USA, 2009, pp. 1-7.

[22] A. A. M. Saleh, "Islands of transparency-an emerging reality in multiwavelength optical networking," in Proc. IEEE/LEOS Summer Topical Meeting on Broadband Opt. Net. and Tech., July 1998, p. 36.

[23] A. Saleh, "Transparent optical networking in backbone networks," in Optical Fiber Communication Conf., Baltimore, MD, USA, 2000, ThD7.

[24] G. Rizzelli, F. Musumeci, M. Tornatore, G. Maier, and A. Pattavina, "Wavelength-aware translucent network design," in $O p$ tical Fiber Communication Conf., Los Angeles, CA, USA, 2011, OThAA2.

[25] J. Y. Yen, "Finding the K shortest loopless paths in a network," Manage. Sci., vol. 17, pp. 712-716, July 1971.

[26] S. D. Personick, "Receiver design for digital fiber optic communication systems, I,” Bell Syst. Tech. J, vol. 52, pp. 843-874, July-Aug. 1973

[27] Deliverable D2.6 of Nobel Project, Phase 1. Network Simulation Ready for Dynamic Transparent Optical Network (A7.1 part).

[28] M. Yannuzzi, M. Quagliotti, G. Maier, E. Marin-Tordera X. Masip-Bruin, S. Sanchez-Lopez, J. Sole-Pareta, W. Erangoli, and G. Tamiri, "Performance of translucent optical networks under dynamic traffic and uncertain physical-layer information," in Int. Conf. Optical Network Design and Modeling, 2009. ONDM 2009, Braunschweig, Germany, 2009, pp. 1-6

[29] O. Bertran-Pardo, J. Renaudier, G. Charlet, P. Tran, H. Mardoyan, M. Salsi, M. Bertolini, and S. Bigo, "Insertion of $100 \mathrm{~Gb} / \mathrm{s}$ coherent PDM-QPSK channels over legacy optical networks relying on low chromatic dispersion fibres," in IEEE Global Telecommunications Conf., 2009. GLOBECOM 2009, Honolulu, HI, USA, 2009, pp. 1-6.

[30] M. Lefrancois, F. Houndonougbo, T. Fauconnier, G. Charlet, and S. Bigo, "Cross comparison of the nonlinear impairments caused by $10 \mathrm{Gbit} / \mathrm{s}$ neighboring channels on a $40 \mathrm{Gbit} / \mathrm{s}$ channel modulated with various formats and over various fiber types," in Conf. Optical Fiber Communication and Nat. Fiber Optic Engineers Conf., 2007. OFC/NFOEC 2007, Anaheim, CA, USA, 2007, pp. 1-3.

[31] B. Garcia-Manrubia, P. Pavon-Marino, R. Aparicio-Pardo M. Klinkowski, and D. Careglio, "Offline impairment-aware RWA and regenerator placement in translucent optical networks," $J$ Lightwave Technol., vol. 29, no. 3, pp. 265-277, Feb. 2011.

[32] K. Christodoulopoulos, K. Manousakis, and E. Varvarigos, "Offline routing and wavelength assignment in transparent WDM networks," IEEE Trans. Netw., vol. 18, no. 5, pp. 1557-1570, Oct. 2010.

[33] M. Murakami and K. Oda, "Power consumption analysis of optical cross-connect equipment for future large capacity optical networks," in 11th Int. Conf. Transparent Optical Networks, 2009. ICTON '09, Azores, Portugal, 2009, pp. 1-4 